\title{
Disperse Solid-Phase Extraction Cleanup for the Determination of 1-Deoxynojirimycin in Mulberry Leaves with Ultraperformance Liquid Chromatography-Tandem Mass Spectrometry
}

\author{
Lei Zhang $\mathbb{D}^{1,},{ }^{1,2}$ Yiran Zhou $\mathbb{D}^{0},{ }^{3}$ Jing Meng $\mathbb{D}^{3},{ }^{3}$ and Jia Li $\mathbb{D}^{2}$ \\ ${ }^{1}$ Taishan University, Tai'an, China \\ ${ }^{2}$ College of Pharmaceutical, Shandong University of Traditional Chinese Medicine, Jinan, China \\ ${ }^{3}$ Jining Institute for Food and Drug Control, Jining, China
}

Correspondence should be addressed to Yiran Zhou; sfdazyr@163.com and Jia Li; 05000045@sdutcm.edu.cn

Received 20 May 2021; Revised 4 July 2021; Accepted 29 July 2021; Published 9 August 2021

Academic Editor: Xiao-zhi Tang

Copyright (c) 2021 Lei Zhang et al. This is an open access article distributed under the Creative Commons Attribution License, which permits unrestricted use, distribution, and reproduction in any medium, provided the original work is properly cited.

A new determination method of 1-deoxynojirimycin (1-DNJ) in mulberry leaves based on ultraperformance liquid chromatography-tandem mass spectrometry (UPLC-MS/MS) has been developed. Dried and crushed mulberry leaves' sample was extracted by $\mathrm{MeCN}$-water solvent, purified by graphitized carbon black (GCB) and primary secondary amine (PSA) to remove organic acids and pigments, and then analyzed after attenuation and filtration. The calibration curve showed linearity in the concentration range of $10-500 \mathrm{ng} / \mathrm{mL}$, with the correlation coefficient of 0.998 . Recoveries of spiked 1-DNJ at three fortification levels ranged from $94.6 \%$ to $96.4 \%$, with relative standard derivation below $1.2 \%$. Additionally, the matrix effect was assessed as negligible. Compared with methods by gas chromatography (GC) and liquid chromatography (LC) via real sample detection, the proposed method acquired better stability and detection efficiency. These results proved that this method has advantages of simple operation, complete purification, small pretreatment loss, good precision and accuracy, and high determination specificity, which is suitable for massive monitoring and precise quantitation of 1-DNJ in mulberry leaves.

\section{Introduction}

Mulberry branch (Ramulus mori) is a traditional Chinese medicinal herb, and its leaves are the main food of silkworm. It has been demonstrated that the intake of mulberry leaves or their extracts could treat diabetes, and the main active component is 1 -deoxynojirimycin (1-DNJ) [1,2], which is a kind of alkaloid compound with nitrogen atoms. It was found to be a potential $\alpha$-glucosidase inhibitor by effectively suppressing the transformation of carbohydrate in the human body, reducing the sugar content in blood, and inhibiting the rapid rise of blood glucose and insulin secretion after eating [3].

At present, the detection methods of 1-DNJ are mainly based on gas chromatography (GC) and high-performance liquid chromatography (HPLC) [4-6]. It is reported that 1DNJ concentrations in mature leaves varied from 0.1341 to
$1.472 \mathrm{mg} / \mathrm{g}$ of dry leaves among 132 mulberry varieties [7]. However, due to the characteristics of high water solubility and weak absorbance, the methods based on GC and HPLC need suitable derivatization processes [4-9], as well as the method employed gas chromatography-tandem mass spectrometry (GC-MS) [10]. Therefore, the complex pretreatment operation will lead to a long preprocessing time and poor stability.

In recent years, ultraperformance liquid chromatography-tandem mass spectrometry (UPLC-MS/MS) has been widely used in the determination of trace amounts, such as pesticides, veterinary drugs, and mycotoxins $[11,12]$. It has also been employed for the determination of 1-DNJ in mulberry leaves, which can overcome the problem of derivatization in the methods based on GC or HPLC and could acquire lower detection limits [13-15]. However, considering the accuracy and repeatability of the method, how to 
effectively remove pigments such as chlorophyll, organic acids, and other interfering substances which lead to the matrix effect and instrument pollution is an important problem for the detection of pesticide residues in vegetables with dark green color [16], which is also applied to the pretreatment process of mulberry leaves. Some materials for purification were synthesized and found to be effective in removing these matrix interferences. Organic acids could be removed by silica microspheres bonded with amidogen, which was upgraded and replaced by primary secondary amine (PSA), while graphitized carbon black (GCB) has better adsorbing capacity on chlorophyll [16]. Some solidphase extraction (SPE) methods based on these materials have been widely used in the analysis of pesticide residues and pollutants in vegetables and herbal plants [17-19], especially the quick, easy, cheap, efficient, rugged, and safe (QuEChERS) method which was the most popular in this field in recent years [20-22].

In this study, a massive monitoring and precise quantitation method of 1-DNJ in mulberry leaves based on LCMS/MS was established, including (1) determining a suitable column and mobile phase proportion and acquiring optimal instrument parameters for the LC-MS system; (2) determining the optimal extraction, purification, and other pretreatment processes; (3) carrying out the methodology validation and comparing with the existing GC and HPLC methods. It is the first development for the determination of 1-DNJ in mulberry leaves with both LC-MS/MS system and matrix interference purification.

\section{Materials and Methods}

2.1. Chemicals and Solvents. Mulberry leaves were collected from Tai'an (Shandong Province, China). The leaves were harvested, cleaned, air-dried at $80^{\circ} \mathrm{C}$ for 12 hours, ground into powder, and then passed through a 100 -mesh sieve and stored in a drying dish until used.

The reference material of 1-deoxynojirimycin (1-DNJ) was purchased from DASF Biological Co., Ltd. (Nanjing, China) with the purity of $98 \%$. Acetonitrile $(\mathrm{MeCN})$ and methanol (HPLC grade) were obtained from Merck (Darmstadt, Germany), while graphitized carbon black (GCB) and primary secondary amine (PSA) purification powder were purchased from Agela Technologies (Tianjin, China).

2.2. Standard and Sample Pretreatment. Stock standard solutions $(100 \mu \mathrm{g} / \mathrm{mL}): 10 \mathrm{mg}$ of 1-deoxynojirimycin standard was accurately weighted (accurate to $0.1 \mathrm{mg}$ ) into a $100 \mathrm{~mL}$ brown volumetric flask and diluted to volume with methanol. The working solution was prepared in the initial mobile phase $(\mathrm{MeCN}$ : water $=1: 1)$ and diluted to prepare the calibration curves at six concentration points of 500,200, $100,50,20$, and $10 \mathrm{ng} / \mathrm{mL}$. The stock solution was kept at $4^{\circ} \mathrm{C}$ before being used.

200 milligrams of the dried mulberry leaf powder was weighed and put into a $50 \mathrm{~mL}$ centrifuge tube. After $50 \mathrm{~mL}$ of $30 \% \mathrm{MeCN} /$ water solution was added, the sample was sonicated in an ultrasonic bath for $5 \mathrm{~min}$, following centrifugation at $7000 \mathrm{r} / \mathrm{min}$ for $5 \mathrm{~min}$ at $4^{\circ} \mathrm{C}$. Subsequently, $5 \mathrm{~mL}$ of the supernatant was collected into a centrifuge tube with $45 \mathrm{mg}$ PSA and $30 \mathrm{mg}$ GCB added and then centrifuged at $10,000 \mathrm{r} / \mathrm{min}$ for $5 \mathrm{~min}$ after being vortexed for $1 \mathrm{~min}$. Finally, $1.0 \mathrm{~mL}$ of purified supernatant was transferred to a $50 \mathrm{~mL}$ volumetric flask and then diluted to volume with the initial mobile phase solvent. The solution was filtered using a nylon syringe filter $(0.2 \mu \mathrm{m})$ before being injected into the LC-MS system.

It should be mentioned that because negative samples with no 1 -DNJ were unavailable, in the tests for pretreatment discussion, samples detected by the published method [6] were employed as quality control (QC) samples; therefore, the parameter "accuracy" was selected to exhibit the relative detection accuracy in the comparing groups. We thought it was a reasonable solution for this test.

2.3. LC-MS/MS Instrumentation. Samples were analyzed by a set of liquid chromatography-tandem mass spectrometry, equipped with the electrospray ionization source (ESI) and triple quadrupole mass analyzer (ExionLC-Triple Quad 3500 , USA). The chromatographic separation was performed with the SunShell C18 $(4.6 \times 100 \mathrm{~mm}, 2.6 \mu \mathrm{m})$ (ChromaNik, Japan) column using a flow rate of $0.35 \mathrm{~mL} /$ min at $40^{\circ} \mathrm{C}$. Mobile phases A and B of HPLC were deionized water and $\mathrm{MeCN}$, respectively, and a 5 min gradient program was employed: proportion of B was kept at 50\% for $2 \mathrm{~min}$, then increased to $90 \%$ within $0.8 \mathrm{~min}$ and kept for $1.1 \mathrm{~min}$, then suddenly decreased to $50 \%$ within $0.01 \mathrm{~min}$, and kept to the end. The injection volume was $2 \mu \mathrm{l}$. ESI was performed in the positive ion mode with the $550^{\circ} \mathrm{C}$ interface temperature. The gas of curtain and ion sources 1 and 2 was set at 10, 50, and 50 psi, respectively. The capillary voltage was set at $5,500 \mathrm{~V}$ and the declustering potential of the parent ion $(\mathrm{m} / \mathrm{z}=164)$ as $40 \mathrm{eV}$. Four ions with $\mathrm{m} / \mathrm{z}$ as 146.0 , 109.9, 128.0, and 69.0 were adopted as product ions, in which the first one was employed as a quantitative ion, and their collision voltages were $19,22,19$, and $26 \mathrm{eV}$, respectively, while the dwell time of each ion was set as $200 \mathrm{~ms}$.

\section{Results and Discussion}

\subsection{Optimization for Instrument Analysis}

3.1.1. Comparison of the Separation Column. Theoretically, 1-DNJ would be better separated by a column bonded with amidogen because it was employed for the separation of monosaccharides such as glucose and fructose with similar structures in the HPLC system [23, 24], while it was used for $1-\mathrm{DNJ}$ analysis and showed a good result in the previous study [13]. In this study, some types of column were employed and tested, including SunShell C18 $(4.6 \times 100 \mathrm{~mm}$, $2.6 \mu \mathrm{m})$, ZORBAX Eclipse Plus C18 $(2.1 \times 50 \mathrm{~mm}, 1.8 \mu \mathrm{m})$, ACQUITY UPLC ${ }^{\circledR}$ BEH HILIC $(2.1 \times 50 \mathrm{~mm}, 1.7 \mu \mathrm{m})$, and Pinnacle II Amino $(4.6 \times 150 \mathrm{~mm}, 3 \mu \mathrm{m})$. As shown in Figure 1, it was found that the SunShell C18 column can lead to the retention time near $2 \mathrm{~min}$, earlier than the Pinnacle II Amino column but later than other columns, while it could 


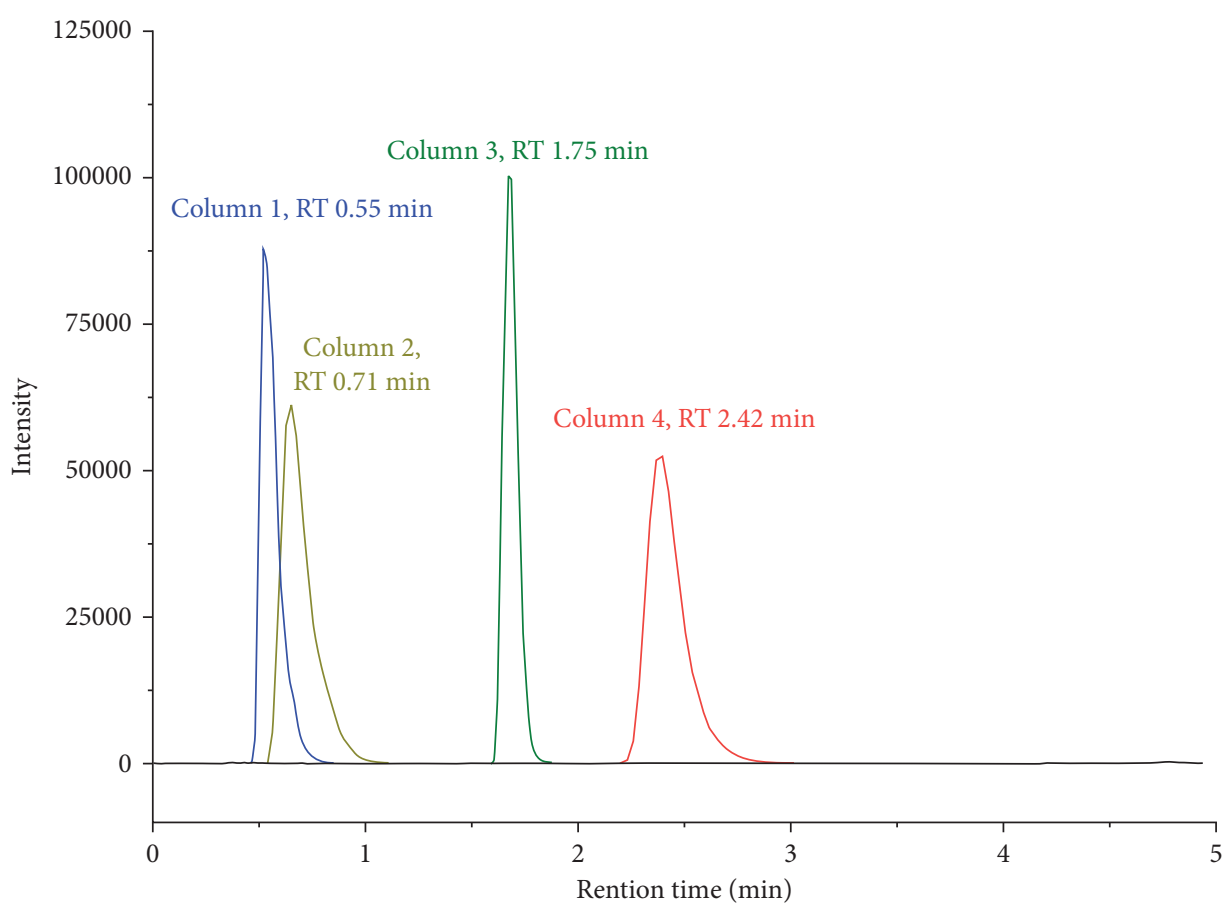

Figure 1: Real sample test chromatograms for the quantitation ion of 1-DNJ with the four types of column $(c=500 \mathrm{ng} / \mathrm{mL})$. Column 1: Agilent ZORBAX Eclipse Plus C18, $2.1 \times 50 \mathrm{~mm} 1.8 \mu \mathrm{m}$, column 2: Waters ACQUITY UPLC ${ }^{\circledR}$ BEH HILIC, $2.1 \times 50 \mathrm{~mm} 1.7 \mu \mathrm{m}$, column 3: SunShell C18, $4.6 \times 100 \mathrm{~mm} 2.6 \mu \mathrm{m}$, and column 4: Pinnacle II Amino, $4.6 \times 150 \mathrm{~mm}, 3 \mu \mathrm{m}$.

acquire a satisfactory peak shape and separation. Thus, SunShell C18 was chosen for the HPLC system in this study.

3.1.2. Mobile Phase and Solvents. Good separation performance and perfect distribution status of target compounds between the stationary and mobile phase would be achieved by appropriate mobile phase and solvents, as well as influence on peak width $[25,26]$. Due to the strong water solubility of 1-DNJ, water would help eluting, while the organic solvent would lead to 1-DNJ retaining on the stationary phase, which differ from distribution characters of other substances. Therefore, the mobile phase proportion and gradient elution procedures must be considered. For the organic solvent, methanol and $\mathrm{MeCN}$ were chosen for the test, and we found that using $\mathrm{MeCN}$ would acquire a better peak shape, smaller column pressure, and better stability of injection. Thus, $\mathrm{MeCN}$ was selected.

Furthermore, the proportion of the initial mobile phase and gradient elution procedure were optimized. It was found that a higher proportion of water at initial would lead to retention time earlier than $1 \mathrm{~min}$ and tend to bring matrix effect, which was unsatisfactory. However, higher ratio of the organic solvent would make matrix interferences be eluted more earlier. Thus, we set up several combinations of them to test the same samples, employing retention time, peak area, and full width at half maximum (FWHM) as parameters. It is shown in Table S1 that, with the increase of the proportion of the organic phase, the retention time decreased, and the peak area value of the target increased, while the FWHM that is closely related to column efficiency showed a gradual decrease. Considering these factors, the mobile phase of water and $\mathrm{MeCN}$ with equal proportion was selected for this method. Moreover, the imino group in the 1-DNJ structure would lead to the cationic characteristic, so it is necessary to provide hydrogen ions for the ionization process by adding formic acid. We also verified the performance while adding $0.1 \%$ formic acid, finding that the response (peak area) increased by $22.6 \%$, and the stability difference was also obvious. Finally, optimal initial of the mobile phase, elution gradient, and solvent for determination were confirmed.

3.1.3. Optimization of Instrument Parameters. Standard solution of 1-DNJ was injected directly into mass spectrometry by using the syringe pump and detected by the ESI source, acquiring a higher response in the positive mode than the negative mode. Strong response of the precursor ion appeared with $\mathrm{m} / \mathrm{z}$ of 164.0 , while product ions of $\mathrm{m} / \mathrm{z} 146.0$, $109.9,128.0$, and 69.0 showed relatively higher response. Thus, in the multiple reaction mode (MRM), based on the difference of response, $\mathrm{m} / \mathrm{z} 146.0$ was reasonable to be applied as the quantitation ion, while the other 3 ions were employed as qualification ions. From the overlaid ion chromatogram shown in Figure 2, we can see that the monitoring parameters can achieve an ideal absolute response value and relative response ratio.

\subsection{Optimization on the Pretreatment Process and Parameters}

3.2.1. Extraction Efficiency of Different Solvents. Mixed $\mathrm{MeCN}$-water was employed as the extraction solvent in this study. We compared the extraction effect of different 


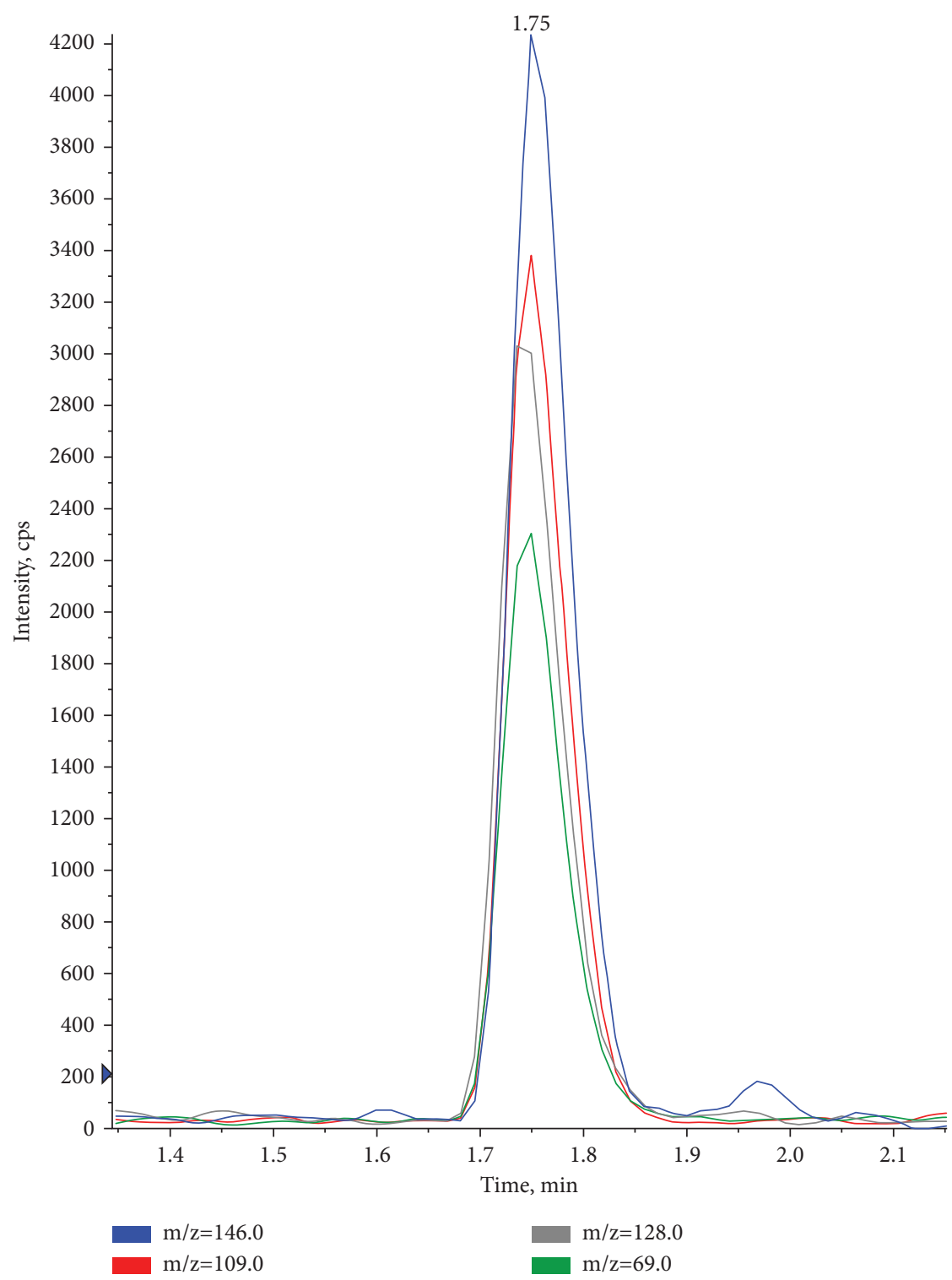

Figure 2: Overlaid chromatogram of four selective ions $(c=50 \mathrm{ng} / \mathrm{mL})$.

proportions of $\mathrm{MeCN}$ in the extraction solvent $(0 \%, 10 \%$, $30 \%, 50 \%, 80 \%$, and $100 \%$ ), as shown in Figure 3 . The highest extraction efficiency was achieved, while the MeCN content was $30 \%$. Because of the strong water solubility of $1-\mathrm{DNJ}$, a higher proportion of water in the extraction solvent was needed, but it still needs some organic solvent to assist extraction.

3.2.2. Research on Purification. There are many coextracts in plant foods, including organic acids and chlorophyll [16]. These substances brought into the LC-MS system will not only cause the contamination of the HPLC system, ion source, and quadrupole mass analyzer but also lead to matrix effect and matrix interference, which will affect the accuracy of determination [27, 28]. Thus, purification for the extraction solvent was set as a key point of this study. Some processes applied in pesticide residue determination were referred [29, 30], including disperse solid-phase extraction (d-SPE) based on GCB and PSA, as well as solid-phase extraction (SPE) based on cation exchange, and comparison was also taken among different cleanup procedures about the performance and efficiency. From Figure 4, we can see that average accuracies by d-SPE, SPE, and no cleanup process were $89.6 \%, 62.4 \%$, and $106.3 \%$, respectively. Although the accuracy of the no cleanup group seems higher, there was an interference peak that did not completely separate with the target peak, which seriously affected quantitation. For the group of SPE, strong water solubility of 1-DNJ leads to considerable loss in the eluting process, and the repeatability was not satisfactory.

Furthermore, dosages of GCB and PSA were discussed. We set the adding amount of both as $10 \mathrm{mg}, 20 \mathrm{mg}, 40 \mathrm{mg}$, and $80 \mathrm{mg}$ in the orthogonal test to compare the parameters of accuracy, stability, and visual purification effect. It was found that accuracy of 1-DNJ improved with increasing 


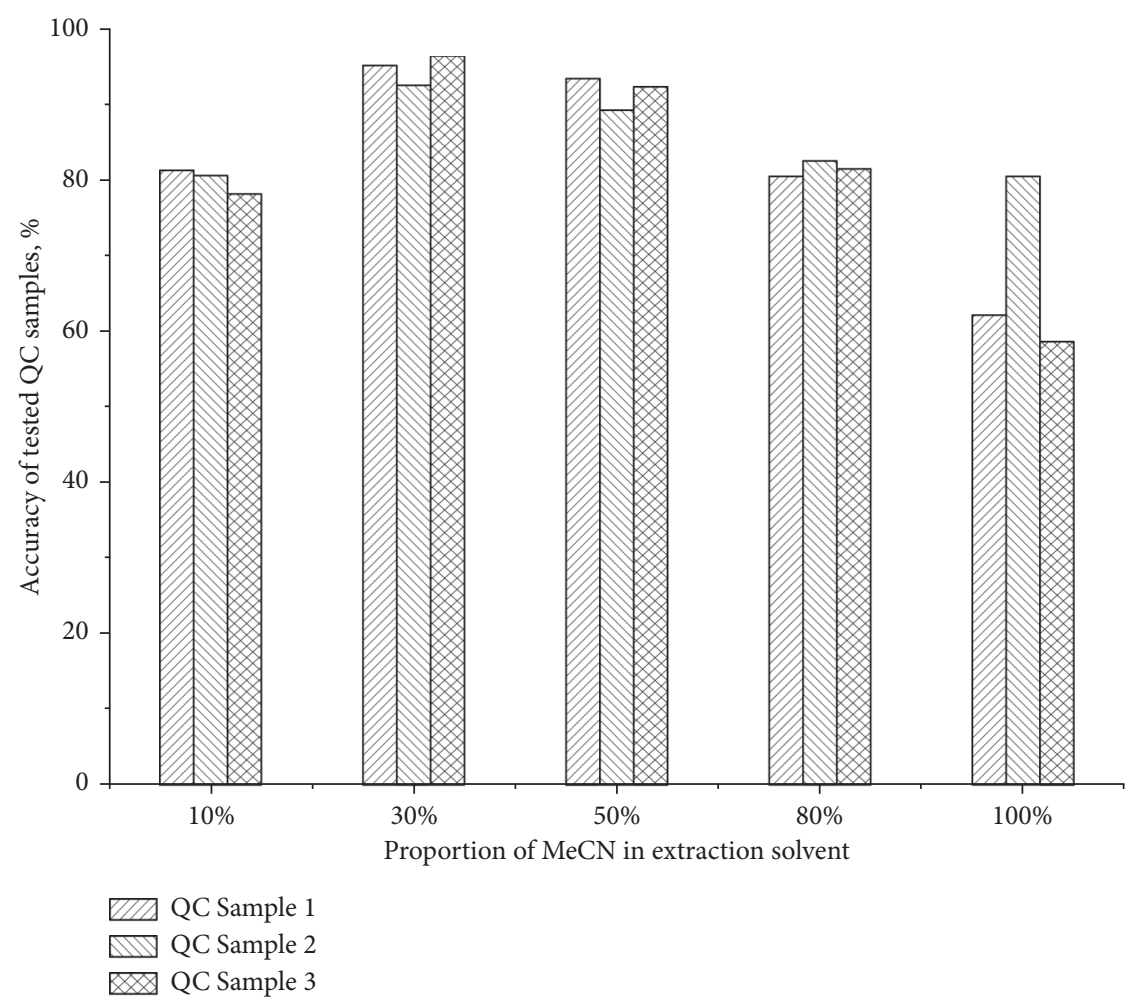

FIGURE 3: Influence of acetonitrile proportion in the solvent on 1-DNJ extraction efficiency.

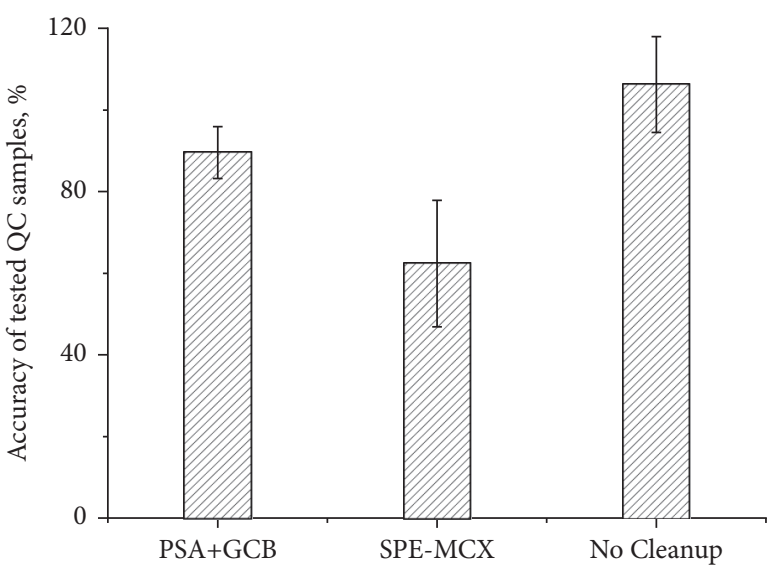

FIGURE 4: Influence of different purification processes on 1-DNJ detection accuracy.

adding amount of PSA, yet it showed little decrease when the adding amount of PSA was more than $40 \mathrm{mg}$. This may be because PSA could effectively adsorb interfering organic acids rather than alkaline compounds such as 1-DNJ, thus reducing matrix interference in mass spectrometry analysis. For GCB, similar regularity was found, but the accuracy significantly decreased while adding more than $40 \mathrm{mg}$. Due to the cyclic planar structure in 1-DNJ molecules, the target would be more or less adsorbed by GCB though there is no conjugated double bond. In terms of visible color, it is shown in Figure 5 that adding more than $20 \mathrm{mg}$ GCB had little effect, indicating that $20-30 \mathrm{mg}$ GCB added could adsorb most chlorophyll in the matrix extraction solvent.
Combining with the accuracy reference of the orthogonal test data in Table S2 and Figure 6, the amount of $45 \mathrm{mg}$ PSA and $30 \mathrm{mg} \mathrm{GCB}$ was determined.

Purification for the extraction solvent will lead to results, the obvious one of which is the change of the acquired ion chromatogram. The extraction ion chromatograms are shown in Figure 7, including purified sample extraction solvent and unpurified one for two samples. It can be seen that the signal of interferences following the peak of 1-DNJ is markedly improved, which would help for the accuracy of quantitation. This is the important reason for employing the purification process.

3.2.3. Dilution Ratio. The contents of $1-\mathrm{DNJ}$ in mulberry leaves were at the level of thousandth $(\mathrm{w} / \mathrm{w})[13,31]$. For LCMS analysis, the optimal linear range was $10-500 \mathrm{ng} / \mathrm{mL}$ in this study. Thus, the dilution ratio of the extraction process was set at about 100 times, which means $0.5 \mathrm{~g}$ mulberry leaves were extracted with $50 \mathrm{~mL}$ extraction solvent and diluted by 50 times during purification and dilution.

3.3. Method Validation. Although the content of 1-DNJ in mulberry leaves was relatively high, the validation for limits of detection and quantitation (LOD and LOQ) was carried out completely in this study. Based on 3 times and 10 times of signal-to-noise ratio, they were acquired as $4 \mathrm{ng} / \mathrm{mL}$ and $10 \mathrm{ng} / \mathrm{mL}$, respectively, and the corresponding method detection limit (MDL) and method quantitation limit (MQL) were $20 \mathrm{mg} / \mathrm{kg}$ and $50 \mathrm{mg} / \mathrm{kg}$, respectively. For standard solvents with concentration higher than $500 \mathrm{ng} / \mathrm{mL}$, 


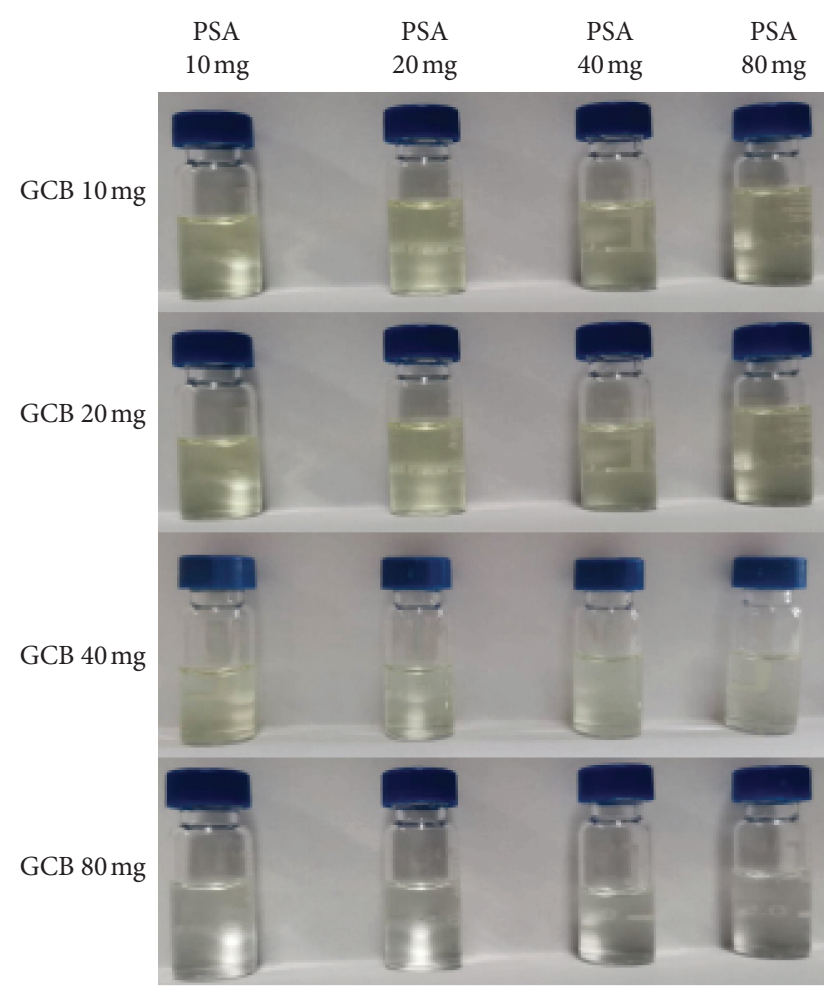

FIGURE 5: Visual color comparison of the orthogonal test for the adding amount of GCB and PSA.

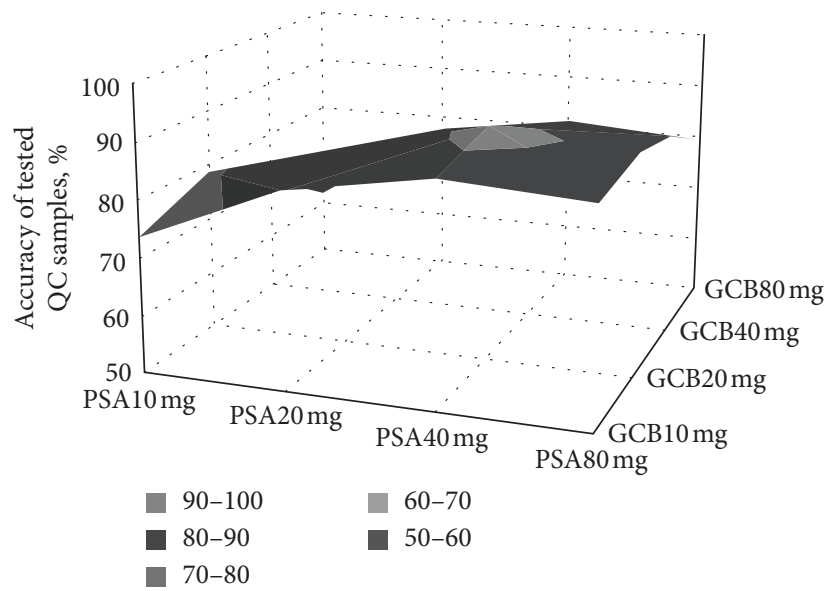

FIgURE 6: Response surface diagram of the data in Table S2.

considerable response attenuation was found, which may be because of competitive soft ionization of 1-DNJ in the ESI source, so the linear range of $10-500 \mathrm{ng} / \mathrm{mL}$ was determined. Six concentration points of 10, 20, 50, 100, 200, and $500 \mathrm{ng} / \mathrm{mL}$ were chosen for the calibration curve, and the linear equation was calculated as $Y=1.3342 e 5 X$, with correlation coefficient as 0.998 . Precision and accuracy were also investigated by spiking in the positive QC sample, the 1DNJ concentration of which was detected by this method as $0.78 \mathrm{mg} / \mathrm{g}$. Spiked concentrations of $1-\mathrm{DNJ}$ were $0.1,0.5$, and $2 \mathrm{mg} / \mathrm{g}$, respectively, with six parallel samples for each concentration. Recoveries for the three levels were $96.4 \%$, $95.7 \%$, and $94.6 \%$, respectively, while the relative standard derivations (RSDs) were $0.9 \%, 1.2 \%$, and $0.9 \%$, respectively. The above validation data were satisfactory, indicating good method applicability.

Considering that negative samples of the mulberry leaf were hard to acquire, much less to prepare matrix standard solution, we investigated the matrix effect. 100 and $500 \mathrm{ng} /$ $\mathrm{mL}$ levels of 1-DNJ were spiked into the test solution of positive samples that had already acquired response peak area to prepare matrix standard solution. The difference value of peak area before spiking and after spiking was employed as the peak area of spiked matrix standard solution. The matrix effect (ME) value was calculated by the equation $\mathrm{ME}=A_{\text {Matrix }} / A_{\mathrm{s}}$, where $A_{\text {Matrix }}$ is the peak area of 


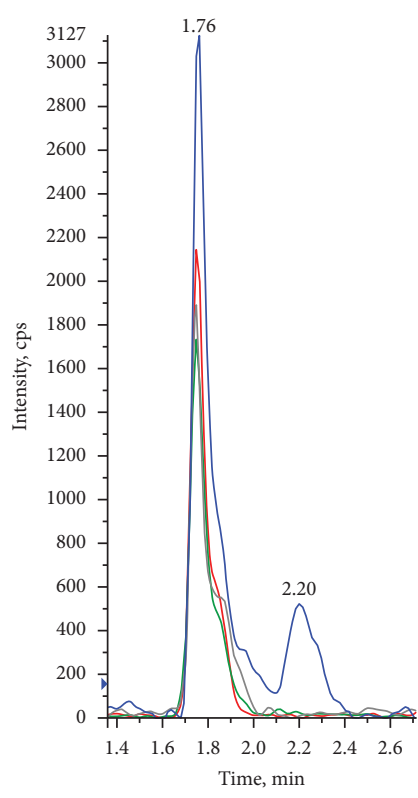

(a)

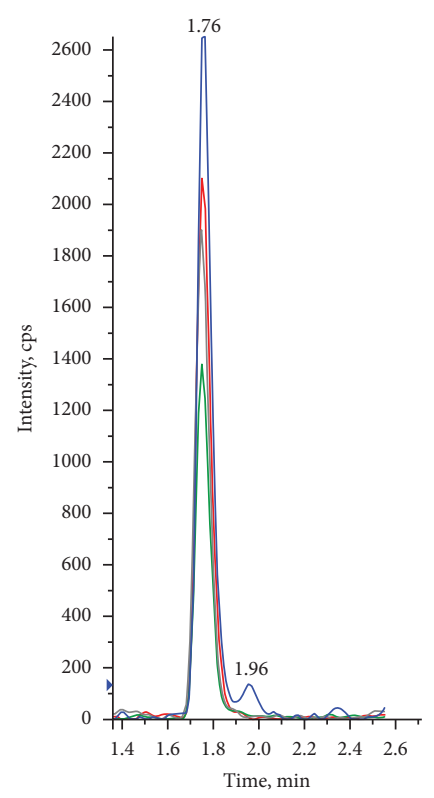

(b)

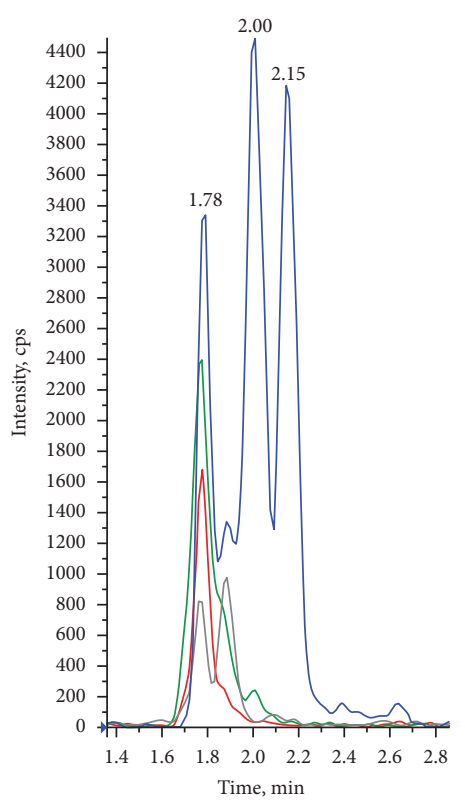

(c)

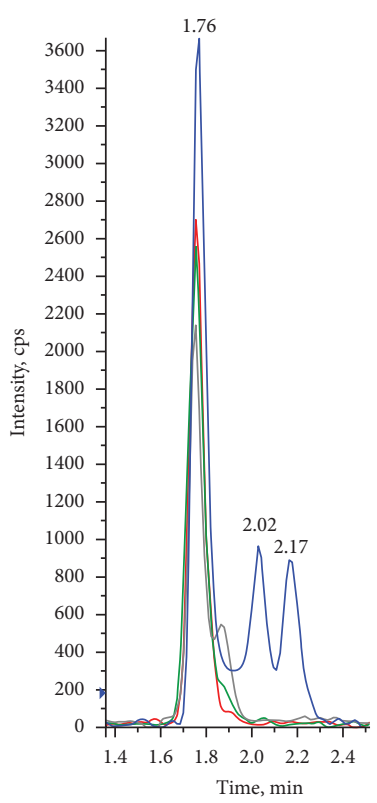

(d)

FiguRE 7: Comparison of chromatograms for purified and unpurified samples ( $c=50 \mathrm{ng} / \mathrm{mL})$. (a) Sample 1, unpurified. (b) Sample 1, purified. (c) Sample 2, unpurified. (d) Sample 2, purified.

spiked matrix standard solution and $A_{\mathrm{s}}$ is the peak area of pure solvent standard solution [19].

The ME was 0.91 and 0.93 , respectively, at the concentration level of 100 and $500 \mathrm{ng} / \mathrm{g}$, which shows little difference. The matrix effect value was considered to be negligible effect at the range of $0.9-1.1$ in the previous study [32]. So, preparing standard solution by initial mobile phase solvent is suitable for this method.

3.4. Investigation of Method Applicability. In this study, thirteen mulberry leaf samples were collected from eight areas of China, and the information is shown in Table S3. All the samples were detected by this method and other two methods based on GC and HPLC, respectively [4, 5]. Every sample was set three parallel tests. The results are also listed in Table S3. There are some different analysis results between the three detection methods. Data of the GC group seem generally lower, and data of the HPLC group seem partially higher and with bad repeatability. The analysis results by this method showed best repeatability and most of them distributed fall in between the other two groups. This is mainly because both methods based on GC and HPLC required derivatization of 1-DNJ; thus, the uncertain efficiency and stability of derivatization lead to the test performance worse than the present study method. For this method, the RSDs of 13 samples ranged from $0.5 \%$ to $1.5 \%$, indicating that this method is relatively mature and stable.

\section{Conclusion}

In this study, a new analysis method for the determination of $1-\mathrm{DNJ}$ in mulberry leaves based on LC-MS was developed. Optimal parameters were determined by testing the instrument analysis condition, while matrix interference was effectively reduced and satisfactory cleanup performance was acquired by studying the procedure process, especially disperse purification based on GCB and PSA. MDL and MQL were verified at 20 and $50 \mathrm{mg} / \mathrm{kg}$, respectively, and this method exhibited good accuracy and precision based on different levels of spiked recovery test, while the matrix effect was found to be negligible. Comparing with the published methods detected by GC or HPLC, this developed method has solved the problems such as poor repeatability and quantitative inaccuracy brought by derivatization and improved the detection efficiency obviously. Furthermore, the developed approach can be used for massive monitoring and precise quantitation of 1-DNJ in mulberry leaves, and it has important application value and innovation significance.

\section{Data Availability}

The data used to support the findings of this study are included within the supplementary information file.

\section{Conflicts of Interest}

The authors declare that they have no conflicts of interest.

\section{Acknowledgments}

This research was funded by the Key Research and Development Program of Shandong Province for the Lu-Yu Science and Technology Cooperation (Grant no. 2019LYXZ023), Science and Technology Development Projects of Tai'an (Grant no. 2019NS087), and TCM Public 
Health Service Special Subsidy in 2019, the National Traditional Chinese Medicine Resources Survey Project.

\section{Supplementary Materials}

Table S1: effect of different mobile phase compositions on the chromatographic peak $(c=100 \mathrm{ng} / \mathrm{mL})$. Table S2: data of the orthogonal test for the adding amount of GCB and PSA $(n=6)$. Table S3: detection results of 1-DNJ from different kinds of mulberry leaf samples via three analysis methods. (Supplementary Materials)

\section{References}

[1] Q. Ge, L. Chen, M. Tang et al., "Analysis of mulberry leaf components in the treatment of diabetes using network pharmacology," European Journal of Pharmacology, vol. 833, pp. 50-62, 2018.

[2] T. Kimura, K. Nakagawa, H. Kubota et al., "Food-grade mulberry powder enriched with 1-deoxynojirimycin suppresses the elevation of postprandial blood glucose in humans," Journal of Agricultural and Food Chemistry, vol. 55, no. 14, pp. 5869-5874, 2007.

[3] K. Gao, C. Zheng, T. Wang et al., "1-Deoxynojirimycin: occurrence, extraction, chemistry, oral pharmacokinetics, biological activities and in silico target fishing," Molecules, vol. 21, no. 11, p. 1600, 2016.

[4] X. C. Zhang, "The study on determination of 1-deoxynojirimycin in mulberry leaves by gas chromatography," MA Dissertation, Northwest A \& F University, Yangling, Xianyang, China, 2007.

[5] X. Zheng, J. Wu, J. He, and Y. Gao, "Determination of DNJ from mulberry leaves by RP-HPLC," Journal of Chinese Institute of Food Science and Technology, vol. 13, no. 1, pp. 188-194, 2013.

[6] J. Tang, Y. Chai, J. Peng et al., "Determination of 1-deoxynojirimycin in mulberry leaf using HPLC-UV with pre-column derivatization," Lishizhen Medicine and Materia Medica Research, vol. 27, pp. 1096-1098, 2016.

[7] X.-Q. Hu, L. Jiang, J.-G. Zhang, W. Deng, H.-L. Wang, and Z.-J. Wei, "Quantitative determination of 1-deoxynojirimycin in mulberry leaves from 132 varieties," Industrial Crops and Products, vol. 49, pp. 782-784, 2013.

[8] J.-W. Kim, S.-U. Kim, H. S. Lee, I. Kim, M. Y. Ahn, and K. S. Ryu, "Determination of 1-deoxynojirimycin in Morus alba L. leaves by derivatization with 9-fluorenylmethyl chloroformate followed by reversed-phase high-performance liquid chromatography," Journal of Chromatography A, vol. 1002, no. 1-2, pp. 93-99, 2003.

[9] E. Nuraniye and D. Emrah, "Determination of 1-Deoxynojirimycin by a developed and validated HPLC-FLD method and assessment of In-vitro antioxidant, $\alpha$-Amylase and $\alpha$-Glucosidase inhibitory activity in mulberry varieties from Turkey," Phytomedicine, vol. 53, pp. 234-242, 2019.

[10] S. Rodríguez-Sánchez, O. Hernández-Hernández, A. I. RuizMatute, and M. L. Sanz, "A derivatization procedure for the simultaneous analysis of iminosugars and other low molecular weight carbohydrates by GC-MS in mulberry (Morus sp.)," Food Chemistry, vol. 126, no. 1, pp. 353-359, 2011.

[11] A. Masiá, M. M. Suarez-Varela, A. Llopis-Gonzalez, and Y. Picó, "Determination of pesticides and veterinary drug residues in food by liquid chromatography-mass spectrometry: a review," Analytica Chimica Acta, vol. 936, pp. 40-61, 2016.

[12] G. Balizs and A. Hewitt, "Determination of veterinary drug residues by liquid chromatography and tandem mass spectrometry," Analytica Chimica Acta, vol. 492, no. 1-2, pp. 105-131, 2003.

[13] N. Nuengchamnong, K. Ingkaninan, W. Kaewruang, S. Wongareonwanakij, and B. Hongthongdaeng, "Quantitative determination of 1-deoxynojirimycin in mulberry leaves using liquid chromatography-tandem mass spectrometry," Journal of Pharmaceutical and Biomedical Analysis, vol. 44, no. 4, pp. 853-858, 2007.

[14] X. Wang, H. Ouyang, Y. Feng, T. Dou, F. Bo, and J. He, "Method of LC-MS/MS for determination of 1-deoxynojirimycin from mulberry leaves of different habitats," Journal of Liaoning University of TCM, vol. 16, no. 12, pp. 60-62, 2014.

[15] B. Xu, D.-Y. Zhang, Z.-Y. Liu et al., "Rapid determination of 1-deoxynojirimycin in Morus alba L. leaves by direct analysis in real time (DART) mass spectrometry," Journal of Pharmaceutical and Biomedical Analysis, vol. 114, pp. 447-454, 2015.

[16] Y.-F. Li, L.-Q. Qiao, F.-W. Li, Y. Ding, Z.-J. Yang, and M.-L. Wang, "Determination of multiple pesticides in fruits and vegetables using a modified quick, easy, cheap, effective, rugged and safe method with magnetic nanoparticles and gas chromatography tandem mass spectrometry," Journal of Chromatography A, vol. 1361, pp. 77-87, 2014.

[17] A. G. Santos, A. C. D. Regis, G. O. Da Rocha, M. D. A. Bezerra, R. M. De Jesus, and J. B. De Andrade, "A simple, comprehensive, and miniaturized solvent extraction method for determination of particulate-phase polycyclic aromatic compounds in air," Journal of Chromatography A, vol. 1435, pp. 6-17, 2016.

[18] Y. Zhou, Y. Lian, X. Sun et al., "Determination of 20 perfluoroalkyl substances in greenhouse vegetables with a modified one-step pretreatment approach coupled with ultra performance liquid chromatography tandem mass spectrometry (UPLC-MS-MS)," Chemosphere, vol. 227, pp. 470479, 2019.

[19] J.-N. Chen, Y.-J. Lian, Y.-R. Zhou et al., "Determination of 107 pesticide residues in wolfberry with acetate-buffered salt extraction and Sin-QuEChERS nano column purification coupled with ultra performance liquid chromatography tandem mass spectrometry," Molecules, vol. 24, no. 16, p. $2918,2019$.

[20] M. Á. González-Curbelo, B. Socas-Rodríguez, A. V. HerreraHerrera et al., "Evolution and applications of the QuEChERS method," TrAC Trends in Analytical Chemistry, vol. 71, pp. 169-185, 2015.

[21] M. C. Bruzzoniti, L. Checchini, R. M. De Carlo, S. Orlandini, L. Rivoira, and M. Del Bubba, "QuEChERS sample preparation for the determination of pesticides and other organic residues in environmental matrices: a critical review," Analytical and Bioanalytical Chemistry, vol. 406, no. 17, pp. 4089-4116, 2014.

[22] S. J. Lehotay, K. Maštovská, and S. J. Yun, "Evaluation of two fast and easy methods for pesticide residue analysis in fatty food matrixes," Journal of AOAC International, vol. 88, no. 2, pp. 630-638, 2005.

[23] K. Mustafa, E. Mustafa, K. U. Mustafa, and A. Mehmet, "Comparison of different extraction and detection methods for sugars using amino-bonded phase HPLC," Journal of Chromatographic Science, vol. 41, no. 6, pp. 331-333, 2003. 
[24] National Health and Family Planning Commission of the People's Republic of China (NHFPC), GB 5009.8-2016 National Standard of Food Safety: Determination of Fructose, Glucose, Sucrose, Maltose and Lactose in Food, China Standard Press, Beijing, China, 2016.

[25] L. A. Riddle and G. Guiochon, "Influence of mobile phase gradients on the retention and separation of peptides from a cytochrome-C digest by reversed-phase liquid chromatography," Chromatographia, vol. 64, no. 3-4, pp. 1-7, 2006.

[26] E. Cabo-Calvet, C. Ortiz-Bolsico, J. Baeza-Baeza, and M. C. García-Alvarez-Coque, "Description of the retention and peak profile for chromolith columns in isocratic and gradient elution using mobile phase composition and flow rate as factors," Chromatography, vol. 1, no. 4, pp. 194-210, 2014.

[27] S. Uclés, A. Lozano, A. Sosa, P. Parrilla Vázquez, A. Valverde, and A. R. Fernández-Alba, "Matrix interference evaluation employing GC and LC coupled to triple quadrupole tandem mass spectrometry," Talanta, vol. 174, pp. 72-81, 2017.

[28] H. Stahnke, T. Reemtsma, and L. Alder, "Compensation of matrix effects by postcolumn infusion of a monitor substance in multiresidue analysis with LC-MS/MS," Analytical Chemistry, vol. 81, no. 6, pp. 2185-2192, 2009.

[29] X. Liu, W. Guan, X. Hao, X. Wu, Y. Ma, and C. Pan, "Pesticide multi-residue analysis in tea using d-SPE sample cleanup with graphene mixed with primary secondary amine and graphitized carbon black prior to LC-MS/MS," Chromatographia, vol. 77, no. 1-2, pp. 31-37, 2014.

[30] G. Stubbings, J. Tarbin, A. Cooper, M. Sharman, T. Bigwood, and P. Robb, "A multi-residue cation-exchange clean up procedure for basic drugs in produce of animal origin," Analytica Chimica Acta, vol. 547, no. 2, pp. 262-268, 2005.

[31] X. Q. Shi, C. J. Chen, F. Li et al., "Determination of 1deoxynojimycin content in leaves of mulberry cultivars in Shandong Province," Science of Sericulture, vol. 39, no. 1, pp. 177-182, 2013.

[32] L. Xiang, L. Chen, T. Xiao et al., "Determination of trace perfluoroalkyl carboxylic acids in edible crop matrices: matrix effect and method development," Journal of Agricultural and Food Chemistry, vol. 65, no. 39, pp. 8763-8772, 2017. 